Cahiers de philosophie de l'université de

\title{
La construction logique de la variété espace-temps. Ordre, métrique, causalité
}

Ivahn Smadja

\section{(2) OpenEdition}

1 Journals

Édition électronique

URL : https://journals.openedition.org/cpuc/1371

DOI : 10.4000/cpuc.1371

ISSN : 2677-6529

Éditeur

Presses universitaires de Caen

Édition imprimée

Date de publication : 15 décembre 2008

Pagination : 225-243

ISBN : 978-2-84133-332-5

ISSN : 1282-6545

\section{Référence électronique}

Ivahn Smadja, "La construction logique de la variété espace-temps. Ordre, métrique, causalité », Cahiers de philosophie de l'université de Caen [En ligne], 45 | 2008, mis en ligne le 04 septembre 2020 consulté le 02 février 2023. URL : http://journals.openedition.org/cpuc/1371 ; DOI : https://doi.org/ 10.4000/cpuc. 1371

\section{(c) (†) 8}

Creative Commons - Attribution - Pas d'Utilisation Commerciale 4.0 International - CC BY-NC 4.0 https://creativecommons.org/licenses/by-nc/4.0/ 


\title{
LA CONSTRUCTION LOGIQUE DE LA VARIÉTÉ ESPACE-TEMPS. ORDRE, MÉTRIQUE, CAUSALITÉ
}

\begin{abstract}
Avec A.A. Robb, A.S. Eddington et quelques autres, B. Russell est l'un de ceux qui, dès les années vingt, ont mis en avant la nécessité de distinguer la structure topologique, la structure métrique et la structure causale de la variété espace-temps de la théorie de la relativité. Je me propose dans cet article de montrer comment les thèses traditionnellement imputées à Russell, à savoir l'indépendance de la structure topologique par rapport à la structure métrique et la dérivation de l'intervalle d'espace-temps à partir de la seule structure causale, ont été articulées dans le contexte d'un programme de construction logique de la variété espace-temps à partir d'événements supposés dépourvus de structure spatio-temporelle. La contextualisation de ces thèses russelliennes permettra de mieux comprendre à la fois leur spécificité et leurs limites.
\end{abstract}

\section{Structures de la variété d'espace-temps}

Toutes les théories d'espace-temps supposent une variété espacetemps fondamentale $M$ munie d'un atlas de cartes locales qui permettent d'assigner des coordonnées $<x_{i}>$ dans un voisinage $\Omega(p)$ de chaque point $p$ de la variété (cf. schéma ci-dessous).

Une carte locale ou un système de coordonnées est une application $\phi: A \rightarrow \mathbf{R}^{4}$ qui associe à tout point $p$ d'un voisinage $A$ de $M$ un quadruplets de nombres réels $\left\langle x_{i}(p)\right\rangle$ dans un ouvert de $\mathbf{R}^{4}$. Pour permettre le recouvrement des cartes locales, on suppose que le changement de cartes $\psi \circ \phi^{-1}$ est une application continue et différentiable. On n'appréhende donc les points de la variété $M$ qu'au moyen de coordonnées au changement de cartes près. De cette manière se trouve définie la structure topologique de la variété espacetemps. 


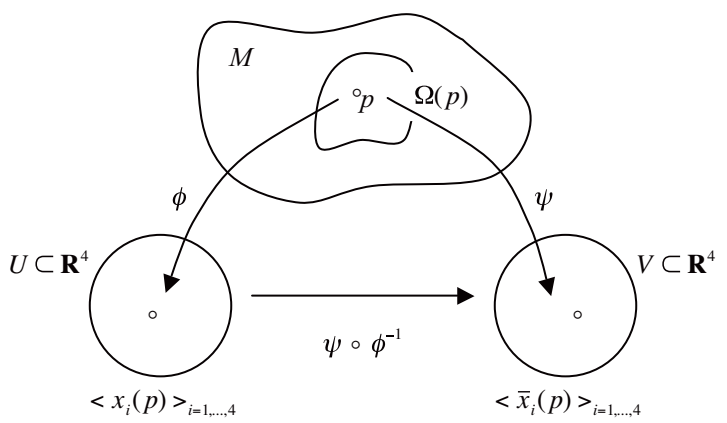

Plus spécifiquement, on considère aujourd'hui que la variété espace-temps est munie de plusieurs structures qui se superposent les unes aux autres et en enrichissent progressivement le contenu ${ }^{1}$ :

- structure topologique,

- structure différentiable,

- structure affine,

- structure métrique.

Pour doter la variété d'une structure différentiable, il faut une condition supplémentaire: si $f$ est une fonction à valeur réelle - par exemple un potentiel, une densité de masse - définie dans un voisinage $\Omega(p)$ de la variété espace-temps $M$, alors $f$ est différentiable si $f \circ \phi^{-1}: \mathbf{R}^{4} \rightarrow \mathbf{R}$ est différentiable pour toute carte locale $\phi$.

Pour pouvoir maintenant représenter la trajectoire d'une particule physique par une courbe dans la variété espace-temps, nous devons nous donner une application continue et différentiable $\sigma: I \rightarrow M$ où $I$ est un intervalle réel, c'est-à-dire paramétrer la courbe de M. Par analogie avec les courbes de l'espace, on définit alors le vecteur tangent $T_{\sigma}\left(t_{0}\right)$ à la courbe en $p$ relativement à la carte $\phi$ comme le quadruplet de nombres réels

$<\frac{d x_{i}(\sigma(t))}{d t}\left(t_{0}\right)>_{i=1, \ldots, 4}$

qui sont les composantes du vecteur $T_{\sigma}\left(t_{0}\right)$ dans le système de coordonnées $^{2}<x_{i}>$. Nous pouvons donc attacher à chaque point un

1. Nous nous référons ici à l'exposé synthétique des différentes structures de l'espace-temps que l'on trouve dans Friedman 1983, chap. II, $\mathbb{\S} 1:$ : General Properties of Space-Time Theories ", p. 32-46.

2. En fait, les composantes du vecteur $T_{\sigma}\left(t_{0}\right)$ changent lorsque l'on change de système de coordonnées, de sorte qu'il serait plus exact de définir le vecteur tangent comme une fonction qui à tout système de coordonnées $\left\langle x_{i}>\right.$ associe un quadruplet $<\left(d x_{i}(\sigma(t)) / d t\right)\left(t_{0}\right)>_{i=1, \ldots, 4}$. 


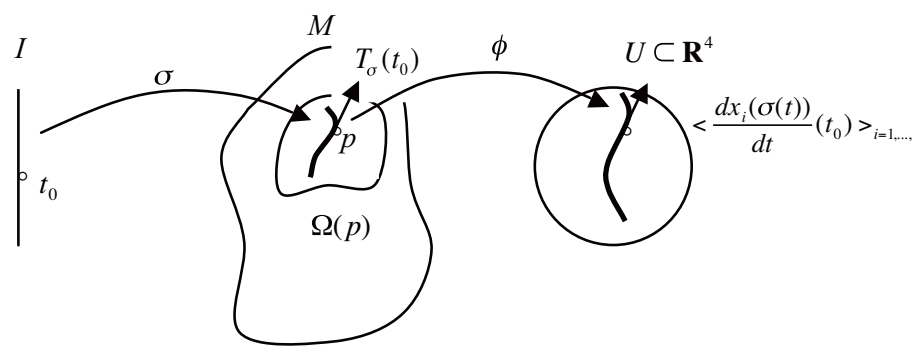

Intervalle de $\mathbf{R}$

Variété espace-temps $M$

Voisinage de $\mathbf{R}^{4}$

vecteur tangent pour chaque courbe $\sigma$. L'ensemble des vecteurs $T_{\sigma}\left(t_{0}\right)$ pour toutes les courbes $\sigma$ passant par $p$ en $t_{0}$, i. e. telles qu'on ait $\sigma\left(t_{0}\right)=p$, est appelé espace tangent en $p$, noté $T_{p}(M)$. Pour pouvoir décrire la forme des courbes dans l'espace-temps, nous devons rendre compte des variations du vecteur tangent lorsque nous parcourons la courbe d'espace-temps et par conséquent nous devons comparer les directions des vecteurs tangents d'un point à un point voisin. Précisons la manière dont nous pouvons procéder à cette comparaison. Soit une courbe et un champ de vecteurs $X(p)$ supposé continu et différentiable, où chaque $X(p)$ est attaché à chaque point $p$ de $\sigma$ et appartient donc au plan tangent $T_{p}(M)$ en $p$. On introduit alors une connexion affine ou une dérivée covariante, notée $\nabla_{T_{\sigma}} X$, de sorte que $\nabla_{T_{\sigma}} X(q)$ est le vecteur qui correspond à la variation du vecteur $X$ au point $q$ lorsqu'on se déplace dans la direction du vecteur $T_{\sigma}$. Grâce à un tel opérateur $\nabla_{T_{\sigma}} X$, nous pouvons coordonner les différents espaces tangents attachés aux points successifs d'une même courbe en suivant de l'un à l'autre la variation d'un vecteur donné. La condition $\nabla_{T_{\sigma}} X=0$ permet ainsi par exemple de déplacer parallèlement le vecteur $\mathrm{X}$ d'un point de la variété à un point voisin dans la direction de $T_{\sigma}$; tandis que $\nabla_{T_{\sigma}} T_{\sigma}=0$ signifie que la courbe ne change pas de direction, ce qui est la définition des géodésiques. Une variété espacetemps munie d'une connexion affine est ainsi dotée d'une structure affine.

On définit enfin une structure métrique sur la variété espacetemps en choisissant un tenseur métrique riemannien $g(X, Y)$ défini sur l'espace tangent $T_{p}(M)$ et correspond à la forme fondamentale $d s^{2}=g_{i j} d x_{i} d x_{j}$. La donnée des $g_{i j}$ permet de calculer les distances par intégration de l'intervalle d'espace-temps, elle détermine donc une notion métrique de géodésique qui doit coïncider avec la notion affine de géodésique. La structure métrique induit 
une structure affine ${ }^{3}$, mais la structure métrique n'est pas réductible à cette dernière.

\section{Constructions logiques}

La "maxime suprême de la philosophie scientifique» consiste selon Russell à toujours substituer, lorsque c'est possible, aux entités inconnues dont infère l'existence des constructions à partir d'entités connues ${ }^{4}$. Selon ce précepte méthodologique, la plupart des objets dont traite la science seraient des "fictions logiques » susceptibles de définition à partir d'éléments plus fondamentaux. Comme les classes en logique, les nombres cardinaux, les points à l'infini de la géométrie projective en mathématiques, les entités de la physique ne feraient donc pas partie de «l'équipement ultime du monde». Dans le domaine des mathématiques pures, les Principia Mathematica visaient à établir que toutes les propositions mathématiques peuvent être déduites, et toutes les entités qu'elles comprennent définies, à partir des constantes, prémisses et principes de la logique. Mais cette méthode est, aux yeux de Russell, susceptible d'être étendue aux théories physiques dont les propositions contiennent, outre les termes logiques, un certain nombre de termes empiriques.

La théorie du symbole incomplet des Principia généralise la théorie des descriptions définies dont le procédé d'élimination des énoncés du langage ordinaire constituait la clef de voûte ${ }^{5}$. Prenons l'exemple des classes. Des expressions de notre langage comme «les habitants de Londres", "les éternels maris", etc. dénotent apparemment des collections mais ne sont pas en fait des notions primitives et peuvent être remplacées par des constructions logiques.

Il faut en chercher une définition qui donnera un sens aux propositions dans l'expression verbale ou symbolique desquelles figurent des mots, ou des symboles, qui en apparence représentent des classes, mais aussi une définition telle que la signification donnée à ces propositions ne comporte aucune référence à des classes; une ana-

3. On obtient les coefficients de Christoffel $\Gamma_{i j}^{k}$ à partir des composantes du tenseur métrique $g_{i j}$.

4. Schilpp 1989, p. 319: «Whenever possible, substitute constructions out of known entities for inferences to unknown entities".

5. Cf. Russell 1910, ${ }^{\star} 14.01$. L'équivalence logique $P((t x)(R x)) \equiv_{\text {def }} \exists x(\forall y(R y \leftrightarrow y=x) \& P x)$ permet, comme on sait, d'éliminer les descriptions du type "le $x$ tel que $R x$ ", i.e. $(\iota x)(R x)$. 
lyse correcte doit donc éliminer toute mention de classes. Nous pourrons dire alors que les symboles de classes sont seulement des outils commodes, non des symboles qui représenteraient des objets appelés "classes"; et qu'en réalité les classes, comme les descriptions, sont des fictions logiques, ou comme nous disons aussi, des symboles incomplets ${ }^{6}$.

Le mécanisme de traduction mis au point par Russell permet ainsi de passer des énoncés qui portent explicitement sur des classes aux énoncés correspondants qui ne mentionnent que certaines propriétés que possèdent les individus des classes supposées ${ }^{7}$. La méthode des constructions logiques peut toutefois être étendue selon le schéma général suivant :

Soit $S_{1}$ un énoncé, employé dans un contexte défini $T_{1}$, qui contient explicitement l'expression " $C$ ", telle que cette expression symbolise une entité $C$; en d'autres termes, « $S_{1}$ " devrait normalement énoncer quelque chose à propos de $C$. Dans quelles circonstances eston fondé à considérer que $C$ est une "construction logique " ou une "fiction logique"? Supposons qu'il existe un ensemble d'entités $a_{1}$, $a_{2}, a_{3}, \ldots$ et un ensemble de relations $R_{1}, R_{2}, R_{3}, \ldots$, qu'un énoncé « $S_{2}$ " puisse être construit qui fasse mention de ces entités et de ces relations, mais ne contienne pas l'expression "C ", et supposons enfin que dans le contexte $T_{1}$, l'énoncé " $S_{2}$ " soit logiquement équivalent à l'énoncé " $S_{1}$ ".

Si toutes ces conditions sont satisfaites, alors $C$ est une construction logique à partir des entités et des relations spécifiées. Remarquons que, comme dans le cas simple des descriptions définies, l'énoncé " $S_{2}$ " ne peut pas en général être obtenu à partir de " $S_{1}$ " simplement en remplaçant l'expression " $C$ " par une expression plus complexe sans modifier la structure d'ensemble de " $S_{1}$ ". La

6. Russell [1918] 1991, p. 337.

7. Cf. Schilpp, p. 323. Puisque deux propriétés différentes peuvent déterminer la même classe, extensionnellement, comme par exemple "x est humain" et "bipède sans plume", le mécanisme de traduction ne doit pas privilégier une propriété plutôt qu'une autre si nous devons respecter le sens de la proposition initiale. La procédure générale de Russell peut-être illustrée par l'exemple suivant. L'énoncé "la classe des points dans un plan compte autant d'éléments que la classe des droites dans un plan" peut être traduit comme ceci : "Il existe au moins deux propriétés telles que l'une d'elles est formellement équivalente à la propriété d'être un point dans un plan, et l'autre formellement équivalente à la propriété d'être une ligne dans un plan, et telle que pour tout individu qui a la première propriété il y ait exactement un individu qui ait la seconde propriété, et inversement.» 
formulation de " $S_{2}$ " requiert en général une refonte complète de " $S_{1}{ }^{8}$.

Le bénéfice philosophique de ce genre de traductions consiste alors en ce qu'elles permettent de couper court aux hypothèses ontologiques auxquelles nos formes d'expression semblaient nous contraindre.

Si nous trouvons le moyen de traiter les classes comme des fictions symboliques, nous augmentons la sécurité logique de notre position, puisqu'à la fois nous évitons l'hypothèse de l'existence des classes, et l'hypothèse opposée selon laquelle il n'y a pas de classes. Simplement nous nous abstenons des deux. C'est là une application du rasoir d'Occam: «ne pas multiplier les entités sans nécessité». Quand nous refusons d'affirmer l'existence des classes, cela ne veut pas dire que nous décidons dogmatiquement qu'il n'y en a pas. Nous restons simplement agnostiques à leur égard; nous disons comme Laplace: «je n'ai pas besoin de cette hypothèse " ${ }^{9}$.

En physique aussi, la méthode des constructions logiques pourrait permettre de faire jouer le rasoir d'Occam. Supposons par exemple qu'on ait affaire à un ensemble de propositions portant sur la structure de l'atome d'hydrogène, rien n'oblige à supposer qu'il y ait réellement une entité E correspondant au terme d'« électron" que ces propositions mentionnent. Une analyse logique alternative est possible qui permettrait, entre autres avantages, d'éviter toute présupposition quant à la localisation des électrons. Nous dirons que deux événements $x$ et $y$ ont entre eux la relation $R$, si intuitivement ce sont deux événements dans la "biographie» de l'électron $E$. La $R$-famille de $x$ consiste alors en $x$ et tous les termes qui ont avec $x$ la relation $R$ et tous les termes avec lesquels $x$ a la relation $R$ et l'on a la traduction suivante.

Si $x$ appartient au domaine de $R$, "l'électron auquel $x$ appartient " signifiera la relation $\mathrm{R}$ avec son domaine limité aux termes appartenant à la $R$-famille de $x$... Pour pouvoir mentionner un électron particulier, nous devons être capable de mentionner un événement qui lui est connecté, par exemple la scintillation lorsqu'il touche un écran donné. Ainsi au lieu de dire «l'événement $z$ est arrivé à l'électron $E$ ", nous dirons simplement "l'événement $z$ est arrivé à l'élec-

8. Cf. Schilpp, p. 324.

9. Russell 1991, p. 341. 
tron auquel $x$ est arrivé», ou plus simplement « $z$ appartient à la $R$ famille de $x \|^{10}$.

Il y a donc en quelque sorte une neutralité ontologique de la méthode des constructions logiques et le philosophe peut s'en tenir à une forme d'agnosticisme face aux entités abstraites et grandeurs inobservables de la physique. Pour autant, il ne s'agit nullement pour Russell d'appliquer dogmatiquement les méthodes de la logique mathématique aux énoncés de la physique, mais au contraire de prendre en compte les théories physiques dans leur complexité pour en déterminer d'abord la structure déductive interne. C'est en effet seulement sur la base de cet examen rigoureux qu'il devient possible d'élaborer une interprétation satisfaisante des théories au moyen de constructions logiques à partir du matériau fondamental que sont les événements ${ }^{11}$.

La philosophie de la physique de Russell concerne principalement la théorie de la relativité, restreinte et générale, quoique les avancées de la théorie quantique soient aussi prises en compte dans L'analyse de la matière (1927), avec prudence toutefois compte tenu du caractère novateur et incertain des résultats les plus récents à cette date. L'objectif essentiel que s'assigne Russell est donc de proposer une reconstruction logique de la variété espace-temps à partir des "événements", en en considérant les différentes structures topologiques, métrique et causale.

\section{Points d'espace-temps}

La première étape du programme logico-philosophique russellien consiste à proposer une construction des points d'espace-temps qui permette de rendre compte de la structure topologique de la variété espace-temps, ou comme dit Russell de sa structure d'ordre spécifique. La méthode de Whitehead pour construire les points, dite méthode des "séries inclusives", consistait à définir un point comme la collection de tous les volumes qui le contiennent. Certaines subtilités sont requises pour éviter que cette définition soit

10. Russell [1927] 1992, p. 287.

11. Sur cette notion d'événement dans la philosophie de la physique de Russell, cf. dans ce même volume, "Structure versus substance. Théories physiques et réalisme structural selon Russell", p. 151. 
circulaire et pour distinguer les ensembles de volumes ayant un point en commun des ensembles de volumes ayant une ligne ou une surface en commun. Quoi qu'il en soit, chaque événement inclurait selon Whitehead d'autres événements et serait inclus dans d'autres événements. Il n'y aurait donc ni limite inférieure ni limite supérieure à la taille des événements. Russell conteste tour à tour ces deux points. Il doit en effet selon lui y avoir une limite supérieure pour une raison qui tient à la définition même de l'«événement" qu'il propose.

Je n'irai pas jusqu'à dire qu'un "événement» ne doit pas avoir de structure. Je supposerai seulement que, quelle qu'elle puisse être, la structure qui pourrait lui être imputée ne relève ni de la physique ni de la psychologie; en d'autres termes, que ses parties, si jamais il en a, n'ont pas de relations à d'autres objets susceptibles d'être distinguées scientifiquement. Quand le mot «événement» est utilisé en ce sens, il est clair qu'aussi loin que nous porte notre expérience, aucun événement ne dure plus de quelques secondes au plus. Il n'y a pas de raison a priori qui expliquerait pourquoi il en est ainsi, c'est simplement un fait empirique ${ }^{12}$.

Si en revanche, il doit aussi y avoir aussi une limite inférieure, c'est que cette hypothèse permet une construction plus satisfaisante, et qu'il faut juger la méthode à ses fruits. Levons toutefois une ambiguïté. Le fait que les événements aient en quelque sorte une extension spatio-temporelle minimale ne préjuge en rien de la structure continue ou discontinue de l'espace-temps. Même en supposant qu'il y a une limite inférieure, que par exemple tout événement remplit au moins un cube quadridimensionnel - c'est-à-dire un centimètre cube durant le temps que met la lumière à parcourir un centimètre - on pourrait néanmoins parfaitement construire un espace-temps continu, grâce à certains axiomes judicieusement choisis.

Esquissons à grands traits cette méthode russellienne de «recouvrement partiel " [partial overlapping]. Partons d'une intuition simple dans le cas d'événements unidimensionnels. Dans la musique orchestrale, toutes les notes jouées concurremment à un instant donné par les différents instruments se chevauchent et sont pour ainsi dire deux à deux co-présentes. Il est alors assez naturel de chercher à définir chaque instant par la classe des événements co-

12. Russell [1927] 1992, p. 294. 


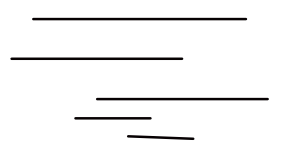

fig. 1

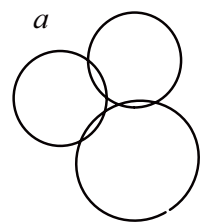

fig. 2

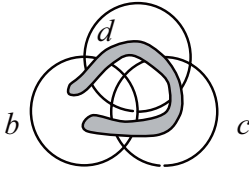

fig. 3

présents. Russell abstrait de ce cas simple une notion générale de "co-présence» entre événements qui puisse être adéquate aux événements de plus d'une dimension, et identifie pour cela les conditions que devrait satisfaire un groupe d'événements pour définir un point d'espace-temps :

(1) Deux événements quelconques du groupe doivent être coprésents.

(2) Aucun événement en dehors du groupe n'est co-présent avec chaque événement du groupe.

Mais ces conditions ne valent plus lorsque l'on passe de la musique orchestrale (cf. fig. 1) à un nombre de dimensions plus grand comme le montre le diagramme ci-dessus (cf. fig. 2). Dès la dimension deux, les événements peuvent être deux à deux co-présents sans avoir d'intersection commune à tous. On pourrait alors chercher à amender la définition en modifiant la première condition et en exigeant que pour des événements de dimension deux,

(1') Trois événements quelconques du groupe doivent être coprésents.

Dans cette phase d'exploration préalable, raisonnons informellement sur des sphères censées symboliser provisoirement les événements, et autorisons leur déformation; nous pourrions alors avoir une configuration où quatre sphères "co-présentes" trois par trois n'auraient cependant aucune région en commun (cf. fig. 3). C'est donc qu'il y a des déterminations de nature quasi-topologique qui, avant même la constitution de l'objet variété espace-temps, imposent certaines contraintes à la définition de la "co-présence" entre événements. Russell se propose en effet de définir les "points» de la variété espace-temps comme classes d'événements satisfaisant certaines conditions, et les "voisinages" comme classes de "points". Mais il est nécessaire de déterminer en amont quelles sont les propriétés que les événements doivent vérifier pour qu'une telle construction 
soit possible. Aussi convient-il de distinguer deux types de considérations topologiques: (1) la topologie de la variété espace-temps, postérieure à la construction des "points " à partir des événements et (2) une sorte de "proto-topologie» des événements qui conditionnerait cette construction même en imposant une certaine définition de la "co-ponctualité " et dont le modèle des sphères dans l'espace euclidien permettrait de cerner les traits essentiels.

L'illustration suivante servira à introduire le problème. Considérons un espace numérique euclidien tridimensionnel, c'est-à-dire la variété de tous les triplets ordonnés $(x, y, z)$ avec la définition usuelle de la distance. Considérons dans cet espace toutes les sphères d'un rayon donné et dont les centres ont des coordonnées rationnelles. Le nombre de ces sphères est $\aleph_{0}$. Définissons un groupe de ces sphères comme "co-ponctuel" si quatre sphères quelconques choisies dans ce groupe ont une région en commun; et définissons un groupe co-ponctuel comme "ponctuel" s'il ne peut pas être élargi sans cesser d'être co-ponctuel. Il y a alors une correspondance biunivoque entre les points d'origine de notre espace et les groupes ponctuels de sphères. Par conséquent, les groupes ponctuels forment un espace euclidien. Si les sphères sont toutes déformées par n'importe quelle transformation continue, elles permettront toujours de construire des groupes ponctuels de la même manière, et la variété des groupes ponctuels aura toujours toutes les propriétés topologiques que possède un espace euclidien tridimensionnel. Si nous utilisons cette méthode de construction des points à partir des événements, il faudra donc que nous supposions qu'il y a, dans l'espace ainsi construit, une métrique possible selon laquelle les "points" dont un certain événement est membre forment toujours un volume sphérique. Bien que cette condition soit exprimée en langage métrique, il s'agit en réalité d'une propriété topologique, puisqu'elle n'est pas affectée par la déformation continue. Il doit être possible de l'exprimer en langage non métrique, mais je dois avouer que je manque de la compétence nécessaire pour le faire.

Je propose par conséquent de regarder les événements comme occupant des régions d'espace-temps qui, dans une certaine métrique, sont des sphères, pour autant qu'il s'agisse de leurs dimensions d'espace, et sont comprises entre un certain maximum et un certain minimum pour autant qu'il s'agisse de leur dimension temporelle. La région "occupée" par un événement est la classe des points dont il est membre.

Comme relation fondamentale dans la construction des points, nous prenons une relation à cinq places, dite de "co-ponctualité " qui a lieu entre cinq événements lorsqu'il y a une région qui leur est commune à tous. Un groupe de cinq événements ou plus est dit 
"co-ponctuel» si tout quintuplet choisi dans ce groupe vérifie la relation de co-ponctualité. Un "point» est un groupe co-ponctuel qui ne peut pas être élargi sans cesser d'être co-ponctuel ${ }^{13}$.

Russell utilise alors l'arsenal de la théorie des ensembles pour démontrer l'existence de tels points d'espace-temps. Grâce à l'axiome de Zermelo ${ }^{14}$, il suppose en effet que tous les événements co-ponctuels avec un quintuplet d'événements donné peuvent être bien ordonnés et montre que tout événement appartient au moins à un point d'espace-temps; puis il caractérise les propriétés logiques des points, en recourant aux notations ensemblistes, où $R(\alpha)$ désigne la classe des événements co-ponctuels avec une classe d'événements $\alpha$ :

(1) Complétion: si $\alpha$ est un point, alors $\alpha=R(\alpha)$, puisqu'un point forme une classe qui ne peut pas être élargie sans cesser d'être co-ponctuelle.

(2) Inclusion: si $\alpha$ et $\beta$ sont deux classes d'événements, et si $\alpha \subset \beta$ alors $R(\beta) \subset R(\alpha)$. Mais si maintenant $\beta$ est un point, alors $\beta=R(\beta)$, et donc par transitivité $\alpha \subset R(\alpha)$. par conséquent, un groupe d'événements est co-ponctuel si $\alpha \subset R(\alpha)$.

D’une manière générale, un événement donné appartient à plusieurs points d'espace-temps, lesquels peuvent remplir à leur tour une région d'espace-temps, mais il est faux que toute région de l'espacetemps soit constituée de points qui ont en commun un certain événement.

\section{Ordre d'espace-temps}

À partir de cette définition des points d'espace-temps, Russell cherche à obtenir la structure topologique ou ce qu'il appelle l'«ordre de l'espace-temps".

La variété espace-temps dans la théorie de la relativité générale a un ordre qui n'est pas arbitraire, et qui est reproduit dans tout système de coordonnées légitimes. Cet ordre, il est important de s'en aviser, est purement ordinal, et n'implique aucun élément métrique. Pas plus qu'il n'est dérivable des relations métriques entre les

13. Ibid., p. 299.

14. L'axiome de Zermelo énonce que le continu peut être bien ordonné, ou encore que pour tout ensemble ayant la puissance du continu, il existe un ordre tel que tout sous-ensemble de l'ensemble initial ait un plus petit élément. 
points, lesquelles sont introduites ultérieurement dans la théorie i. e. à partir des «intervalles»"

Les transformations de coordonnées admissibles en analyse tensorielle laissent les relations de voisinage inchangées. En ce sens, les propositions relatives à l'ordre d'espace-temps doivent demeurer vraies, lorsque les configurations de points sont soumises à déformation continue. Russell construit donc la relation d'ordre entre points à partir d'une sorte de feuilletage des événements. Partant d'un événement donné, la classe de tous les événements qui lui sont co-présents constitue une première enveloppe, puis la classe de tous les événements qui ne sont pas co-présents à l'événement initial mais sont co-présents à un événement de la première enveloppe constitue une seconde enveloppe, et ainsi de suite. Les événements pourront alors être répartis en zones, car chaque événement appartiendra à une zone déterminée par rapport à un événement de référence, et deux événements donnés seront séparés par un nombre minimum de pas.

Lorsqu'un événement peut être atteint à partir d'un autre en $n$ pas mais non en $n-1$, nous pouvons regarder les événements intermédiaires comme formant une sorte de route géodésique quantifiée entre les deux événements ${ }^{16}$.

Deux points seront dits "connectés" s'il y a un événement qui leur appartient à tous deux. Sur cette base, une alternative se présente selon que les points sont connectés ou non. Russell distingue en effet un "ordre macroscopique» entre points non connectés, lequel suppose la répartition des points en zones, et un "ordre microscopique» entre points connectés, lequel suppose la définition d'une relation de betweenness entre points d'une même zone.

Pour ce qui est de cette seconde relation d'ordre, l'intuition directrice est très simple si on l'exprime en langage géométrique : le point $\lambda$ est entre les points $\kappa$ et $\mu$, si tout événement qui contient à la fois $\kappa$ et $\mu$ contient aussi $\lambda$, mais tout événement qui contient à la fois $\kappa$ et $\lambda$ ne contient pas nécessairement $\mu$. Ce que Russell couche dans le langage de la théorie des ensembles de la manière suivante :

" $\lambda$ est entre $\kappa$ et $\mu$ » signifie $« \kappa, \lambda, \mu$ sont des points tels que $\kappa \mu$

[i. e. produit logique de $\kappa$ et $\mu$ ] est non nul, et est contenu dans $\lambda$, mais $\kappa \lambda$ n'est pas contenu dans $\mu$ ".

15. Russell [1927] 1992, p. 56.

16. Ibid., p. 304. "a sort of quantized geodesic route between the two events". 


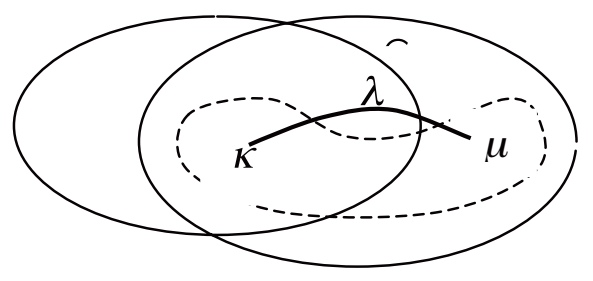

fig. 4

Une objection sérieuse peut toutefois être formulée à l'encontre de cette définition. Si en effet les événements peuvent avoir des formes irrégulières ${ }^{17}$, alors l'intuition sous-jacente devient inopérante (cf. fig. 4). Face à cette difficulté, Russell formule une sorte d'hypothèse de convexité des événements, nécessaire pour que la définition de la relation de betweenness puisse être correcte. La circularité de l'argument ne serait alors qu'apparente car cette notion primitive de protoconvexité des événements serait antérieure à la structure topologique de la variété espace-temps.

Sur la base de cette relation d'ordre, Russell définit un ensemble de points "collinéaire" comme une collection telle que deux points quelconques sont toujours connectés, et trois points quelconques $\alpha, \beta, \gamma$ vérifient soit $\alpha \beta \subset \gamma$, soit $\alpha \gamma \subset \beta$. Une «ligne» est alors un ensemble de points qui (1) est collinéaire, et (2) n'est contenu dans aucun groupe collinéaire plus grand ayant les mêmes extrémités. Cette seconde clause de complétion entre dans la définition de la ligne comme dans celle du point, établissant ainsi un parallélisme entre les deux définitions. L'étape suivante consiste alors à élaborer, grâce à des axiomes appropriés, une définition satisfaisante de l'ordre parmi les points d'un ensemble collinéaire de points, puis à définir, par ajout d'un nouvel axiome, la notion de «limite»: une progression de points collinéaires qui se trouvent tous entre deux points et $\beta$ doit avoir une limite. L'ensemble de la démarche permet de définir les notions topologiques fondamentales à partir des relations logiques entre ensembles d'événements. Il importe de remarquer cependant que Russell n'introduit aucun axiome d'existence quant à la cardinalité de l'espace-temps.

17. L'événement représenté par le tracé en pointillés (cf. fig. 4) met en échec la définition de la relation de betweenness. 
Nous ne savons pas si l'espace-temps est continu ou non. Quoi qu'il en soit il est intéressant d'observer qu'un appareillage initial de $\aleph_{0}$ événements suffit à engendrer un espace-temps continu de points, au moyen des relations de co-ponctualité et d'inclusion logique ${ }^{18}$.

Mais la construction n'est pas encore achevée. Il faut encore définir les "surfaces" et les "régions" de l'espace-temps de la même manière que les "points" et les «lignes", c'est-à-dire au moyen de relations de "co-superficialité", respectivement de "co-régionalité", et de l'inclusion logique. Par exemple, dans le cas des «surfaces»:

(1) Une classe de lignes est dite "co-superficielle» lorsque deux lignes quelconques de la classe s'intersectent, mais qu'il n'y a pas de point commun à toutes les lignes de la classe.

(2) Une «surface» est une classe de lignes co-supeficielles qui ne peut pas être augmentée sans cesser d'être co-superficielle.

On procéderait de manière analogue pour les notions de classe de surfaces "co-régionale» et de "région" d'espace-temps. Russell établit alors que l'espace-temps ainsi construit est une variété topologique au sens de Hausdorff et vérifie pour finir que cette variété topologique est bien de dimension quatre ${ }^{19}$.

Deux remarques peuvent être formulées. La première concerne les rapports entre structure topologique et structure métrique. Russell se propose, nous l'avons vu, de définir l'espace-temps comme variété topologique à partir des événements, mais concernant la structure métrique, il se contente de suggérer une piste qui pourrait permettre de définir une métrique à partir de la topologie, à tout le moins localement ${ }^{20}$, mais rien ne garantit que cette hypothèse de travail puisse être utilement élaborée. L'autre remarque a trait à l'ordre lui-même. Nous avons vu que Russell distingue deux notions d'ordre, "macroscopique", entre événements non connectés, et "microscopique", entre événements connectés, sans que la question de leur liaison soit clairement posée. L'ordre global s'obtientil en ressoudant les ordres locaux, et, si tel est le cas, comment s'opèrent ces soudures? Ces questions sont laissées en suspens.

18. Russell [1927] 1992, p. 311.

19. Ibid. p. 312.

20. Pour réaliser le second volet de ce programme, Russell utilise un théorème de topologie, le théorème d'Urysohn, qui énonce que tout espace topologique satisfaisant à certaines conditions déterminées est métrisable. 


\section{Structure causale}

Si, parallèlement à la causalité extrinsèque des phénomènes quantiques $^{21}$, il y a une causalité intrinsèque incorporée à la géométrie de l'espace-temps, alors la question se pose naturellement de savoir comment il serait possible d'abstraire la structure causale sousjacente à la variété espace-temps. Partant de ce fait que la structure géométrique de l'espace-temps de Minkowski incorpore une théorie non-classique de la structure causale, liée à la relativité de la simultanéité, à la dilatation des temps et à la contraction des distances, Robb (1914) avait clairement posé le problème de savoir si la structure causale de l'espace-temps de Minkowski en comprend en elle-même la géométrie entière, c'est-à-dire aussi bien la structure topologique que la structure métrique ${ }^{22}$.

Russell s'inspire de certaines de ces idées, notamment la notion, importante dans la perspective de Robb, selon laquelle, lorsque deux événements ont entre eux un intervalle de genre espace, il ne peut y avoir aucune relation causale directe entre eux; et, par conséquent, pour deux événements de ce type, s'il doit y avoir inférence de l'un à l'autre, ce devrait être nécessairement par l'intermédiaire d'un "ancêtre causal» commun. Plus fondamentalement, Russell cherche à définir l'intervalle d'espace-temps à partir des relations causales entre événements.

Il y a relation causale à chaque fois que deux événements, ou deux groupes d'événements dont un au moins est co-ponctuel, sont liés par une loi qui permet d'inférer quelque chose à propos de l'un à partir de l'autre. Auparavant, on aurait supposé qu'on pouvait tout inférer à propos du second événement à partir d'un nombre suffisant d'antécédents, mais [du fait de la prise en compte des phénomènes quantiques], nous devons nous satisfaire d'une définition plus modeste sur ce point ${ }^{23}$.

Russell présente les lignes directrices d'une conception d'ensemble unissant structure causale et intervalle puis l'affine ensuite progressivement :

21. Sur ce concept de causalité extrinsèque et son importance pour les phénomènes quantiques, cf. "Structure versus substance. Théories physiques et réalisme structural selon Russell", p. 152-157.

22. Winnie 1977, p. 135.

23. Russell [1927] 1992, p. 368. 
Étant donné deux groupes d'événements co-ponctuels, il peut arriver qu'au moins un membre d'un groupe ait une relation causale à au moins un membre de l'autre groupe; dans ce cas, l'intervalle entre les deux groupes est de genre temps. Si la causalité est affaire de transitions discontinues, on pourrait s'attendre à ce que la grandeur de l'intervalle soit mesurée par le nombre de transitions intermédiaires. Maintenant, il peut arriver qu'aucun membre d'un groupe n'ait de relation causale à quelque membre que ce soit de l'autre groupe, mais que les deux groupes contiennent des membres ayant des relations causales avec un membre d'un troisième groupe. Dans ce cas, l'intervalle sera dit de genre espace, et on pourra là aussi supposer que le nombre de liens intermédiaires détermine la grandeur de l'intervalle ${ }^{24}$.

Les contraintes de la construction conduisent alors à considérer séparément les intervalles de mesure nulle, les intervalles de genretemps et les intervalles de genre espace, car on ne peut pas les définir d'une manière uniforme, notamment parce que les seconds dépendent des premiers.

Si un événement $e_{1}$ est ancêtre causal d'un événement $e_{2}$, Russell propose de considérer toutes les routes causales possibles de $e_{1}$ à $e_{2}$, et de choisir celle qui contient le plus grand nombre d'événements. Il définit alors l'intervalle entre $e_{1}$ et $e_{2}$ comme le nombre des événements qui composent la route causale la plus longue de l'un à l'autre, de sorte que l'on puisse retrouver ainsi l'intervalle de genre temps de la théorie de Minkowski.

Plus généralement, Russell explicite en termes de relations causales toute l'information contenue dans les diagrammes d'espacetemps de Minkowski. Dans le cas des intervalles de genre espace, il part d'une situation idéalisée où deux rayons lumineux sont envoyés de la Terre vers le Soleil et du Soleil vers la Terre, puis réfléchis par des miroirs à leur arrivée et renvoyés vers leur source (cf. fig. 5). Soient alors les deux événements $S$ et $S$ ' que constituent les deux émissions de lumière depuis la Terre et le Soleil respectivement, et soit $T$ l'événement correspondant à la réception sur Terre du rayon solaire émis en $S^{\prime}$.

Supposons d'une part que la route causale la plus longue entre les deux transactions $S$ et $T$ consiste en $n$ événements $e_{1}, e_{2}, \ldots, e_{n}$, et d'autre part qu'un même événement lumineux soit à la fois coprésent à $S$ ' et $T$ (cf. fig. 6). On remarque qu'il ne peut y avoir de

24. Russell [1927] 1992, p. 367. 


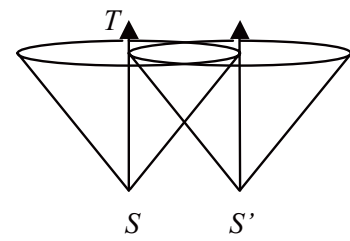

fig. 5

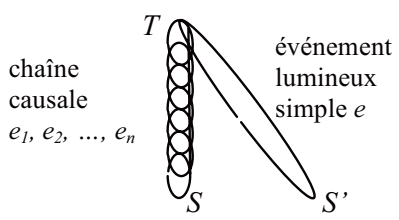

fig. 6

connexion causale de $S^{\prime}$ à $S$, car si tel était le cas, il y aurait une route causale de $S^{\prime}$ à $T$ plus longue que l'événement lumineux $e$, à savoir celle passant par $S$, ce qui est contraire à ce que nous avons supposé. Dans ce type de configuration, on dit alors que l'intervalle entre $S$ et $S$ ' est de genre espace et a même valeur numérique que l'intervalle de genre temps entre $S$ et $T$. On donne ainsi aux intervalles de genre espace, un sens analogue à celui qui peut être déduit de la métrique $d s^{2}=c^{2} d t^{2}-d x^{2}-d y^{2}-d z^{2}$ et qu'expriment les diagrammes de Minkowski, mais exprimé cette fois-ci en termes causaux.

Il reste alors à rendre compte des intervalles de mesure nulle. L'exemple paradigmatique est le battement d'une horloge optique : un rayon lumineux de $A$ à $B$ est réfléchi de $B$ en $A$. Ce cas ne fait intervenir que deux transactions, l'émission et la réflexion du rayon de lumière, mais il s'agit selon Russell d'un seul et même événement, l'"événement lumineux", qui part de $A$ et arrive en $B$, par conséquent il n'y a pas de route causale plus longue et l'intervalle est égal à zéro.

\section{Conclusion}

Si l'on juge le programme russellien de construction logique de la variété espace-temps à l'aune des développements ultérieurs qui ont permis de préciser la superposition de structures topologique, différentiable, affine et métrique, qui caractérisent cette variété, ainsi que leurs relations de dépendance ou d'indépendance ${ }^{25}$, on peut reconnaître à Russell le mérite d'avoir nettement mis en lumière les deux aspects essentiels suivants: (1) la topologie de l'espacetemps ne doit pas être envisagée comme la topologie induite par la

25. Cf. Earman, Glymour et Stachel 1977. 
métrique, et (2) la structure causale est étroitement liée à l'intervalle d'espace-temps.

Mais le travail de Russell sur les fondements de la théorie de la relativité était sérieusement hypothéqué par le fait qu'il fut induit en erreur par certains traits accidentels de l'intervalle, comme par exemple l'assignation d'une valeur nulle aux intervalles entre événements le long d'un même rayon lumineux ${ }^{26}$.

Aussi Russell n'est-il pas parvenu à clairement articuler structure métrique et structure causale du fait d'une interprétation trop littérale de l'intervalle d'espace-temps, mais aussi en raison des présupposés philosophiques liés à la notion d'événement qu'il s'était donnée.

Ivahn SMADJA

Université Denis Diderot - Paris 7

26. Winnie 1977, p. 157. 


\section{Références bibliographiques}

Earman J. S., Glymour C. N. et Stachel J. J. (éd.) (1977), Foundations of Space-Time Theories, Minneapolis, University of Minnesota Press (Minnesota Studies in the Philosophy of Science; vol. VIII).

FRIEDMAN M. (1983), Foundations of Space-Time Theories. Relativistic Physics and Philosophy of Science, Princeton, Princeton University Press.

Robb A. A. (1914), A Theory of Space and Time, Cambridge, Cambridge University Press.

Russell B. (1910), Principia Mathematica, Cambridge, Cambridge University Press, t. I.

Russell B. [1918] (1991), Introduction à la philosophie mathématique, F. Rivenc (trad.), Paris, Payot.

Russell B. [1927] (1992), The Analysis of Matter, Londres - New York, Routledge.

Schilpp P. A. (éd.) (1989), The Philosophy of B. Russell, La Salle, Illinois, Open Court (The Library of Living Philosophers; vol. V).

WINNIE J. A. (1977), "The Causal Theory of Space-Time», in Earman, Glymour \& Stachel (1977), p. 134-205. 